\title{
Diamond Dissolution Forms in Water-Containing and Water-Free Carbonate Melts
}

\author{
Alexander F. Khokhryakov and Yury N. Palyanov \\ Institute of Geology and Mineralogy SB RAS, Novosibirsk, Russian Federation
}

Morphology of most natural diamonds is the result of their dissolution (oxidation) in kimberlitic melt. Since rounded diamonds occur in kimberlites and lamproites it is supposed that the dissolution of natural diamonds took place in ultramaffic carbonate-silicate melts, whose main volatile components were $\mathrm{CO}_{2}$ and $\mathrm{H}_{2} \mathrm{O}$. However, the factors affecting the morphology and preservation degree of diamonds have been still poorly understood. The experiments showed the important role of volatile and first of all of water during the natural dissolution of diamond (Kanda et al., 1977, Khokhryakov, Palyanov, 2000, Kozai, Arima, 2005, Khokhryakov, Palyanov, 2007, Fedortchouk et al., 2007). Among the main components of kimberlitic melts, of particular interest are carbonates. It has been shown that at a pressure of $1 \mathrm{GPa}$ carbonate melts do not react with diamond (Kozai, Arima, 2005, Fedortchouk et al., 2007). However, at higher pressures in the field of thermodynamic stability of diamond carbonate melts are active solvents of carbon and, furthermore, provide diamond crystallization. To elucidate the role of carbonates upon natural diamond dissolution we performed an experimental study on diamond dissolution in volatile-free and $\mathrm{H}_{2} \mathrm{O}$ and/or $\mathrm{CO}_{2}$ containing carbonate melts.

\section{Experimental}

Experiments on diamond dissolution were carried out using a "split-sphere" type multi-anvil apparatus at $\mathrm{P}=5.7-7.5 \mathrm{GPa}, \mathrm{T}=1400-1750^{\circ} \mathrm{C}$. Details on the highpressure sell design, calibration of pressures and temperatures were presented in our previous work (Pal'yanov et al. 2002). Starting materials and several diamond crystals were placed into sealed Pt capsules. No buffer materials were added to the high-pressure cell. Previously we showed that in this case oxygen fugacity in $\mathrm{Pt}$ capsules under experimental conditions is 1-2 logarithmic units lower than CCO buffer (Sokol et al. 2000). For studies we used natural octahedral diamond crystals $0.3-0.8 \mathrm{~mm}$ in size without dissolution features from Udachnaya and Aikhal kimberlite pipes (Yakutia). Starting materials were $\mathrm{Na}_{2} \mathrm{CO}_{3}, \mathrm{CaCO}_{3}$ and natural dolomite. $\mathrm{H}_{2} \mathrm{O}$ and $\mathrm{CO}_{2}$ were added as distilled water, silver oxalate or oxalic acid. The crystals were analyzed with optical and scanning electron microscopes. Etch pits were examined using a double-reflecting interference microscope. Rounded crystals were studied using the photogoniometric method.

\section{Dissolution of diamond in volatile-free carbonate melts.}

The changes in the morphology of diamond crystals upon dissolution in the volatile-free carbonate melts were traced for the crystals weight loss of up to $66 \%$. In short experiments triangle etch pits are found to form on the $\{111\}$ diamond faces. The pits have edges parallel to the contour of octahedral face and are known as positive trigons (Fig. 1).
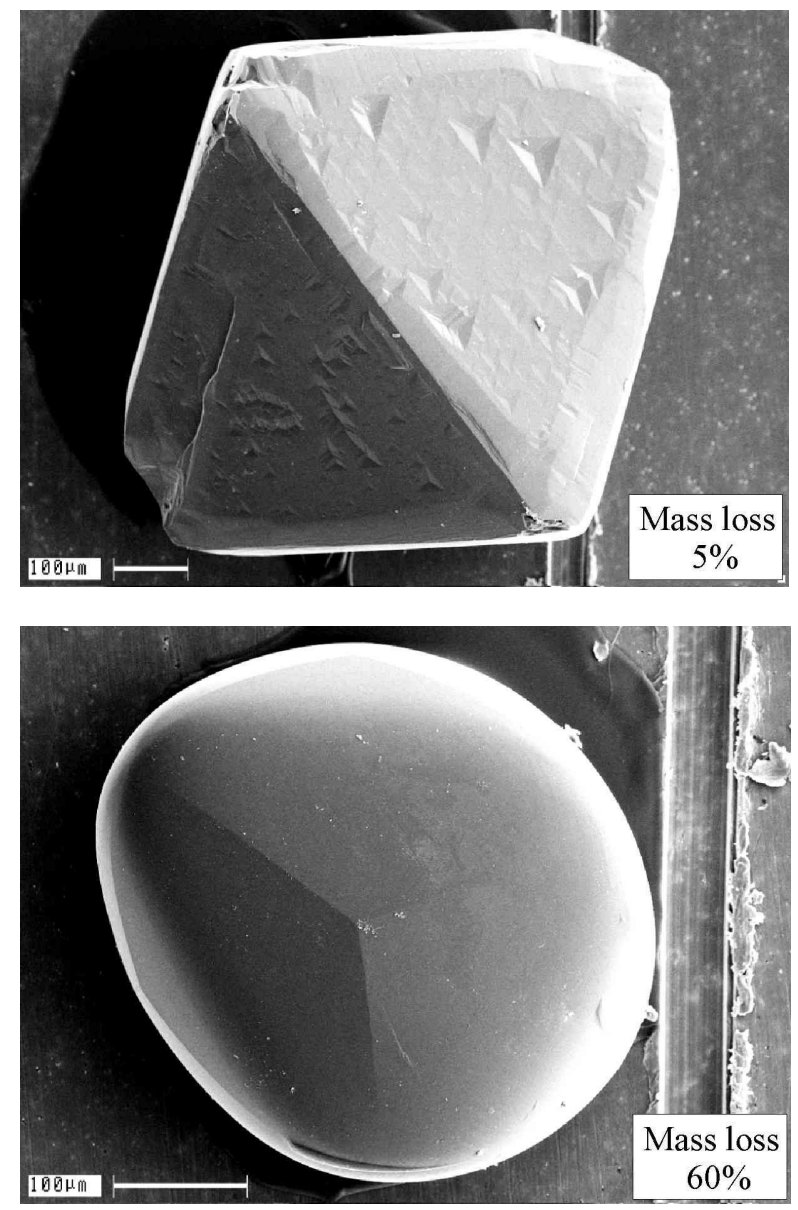

Fig. 1. SEM micrographs of dissolution forms of diamond in volatile-free carbonate melts. 
The inclination of the walls of the pits to the $\{111\}$ faces is $5^{\circ}$ and $13^{\circ}$, that correspond to the $\{655\}$ and $\{855\}$ faces. Beside the etch pits, dissolution surfaces of trigon-trioctahedron are formed along the crystal edges. At the dissolution degree of more than $18-20 \%$ the initial $\{111\}$ faces and etch pits disappear. The surfaces of dissolution forms are for the most part smooth. From the presence and position of the edges of the produced dissolution form it follows that it nearly corresponds to dodecahedroid. However, as opposed to dodecahedroid, the produced dissolution forms do not possess the $<001>$ apexes. The edges propagating from the $<111>$ apexes do not reach the $<001>$ apexes. At further increase in the dissolution degree the crystals progressively attain more rounded ball-shaped form. The edges spreading from the $<111>$ apexes become shorter. For the dissolution degree of more than $60 \%$ the endings of these edges approximately corresponds to the points where perpendiculars to the $\{955\}$ faces emerge (Fig. 1).

\section{Dissolution of diamond in $\mathrm{CO}_{2}$-containing carbonate melts.}

The changes in the diamond crystal morphology upon dissolution in $\mathrm{CO}_{2}$-contaning carbonate melts were traced for the crystals weight loss of up to $29 \%$.
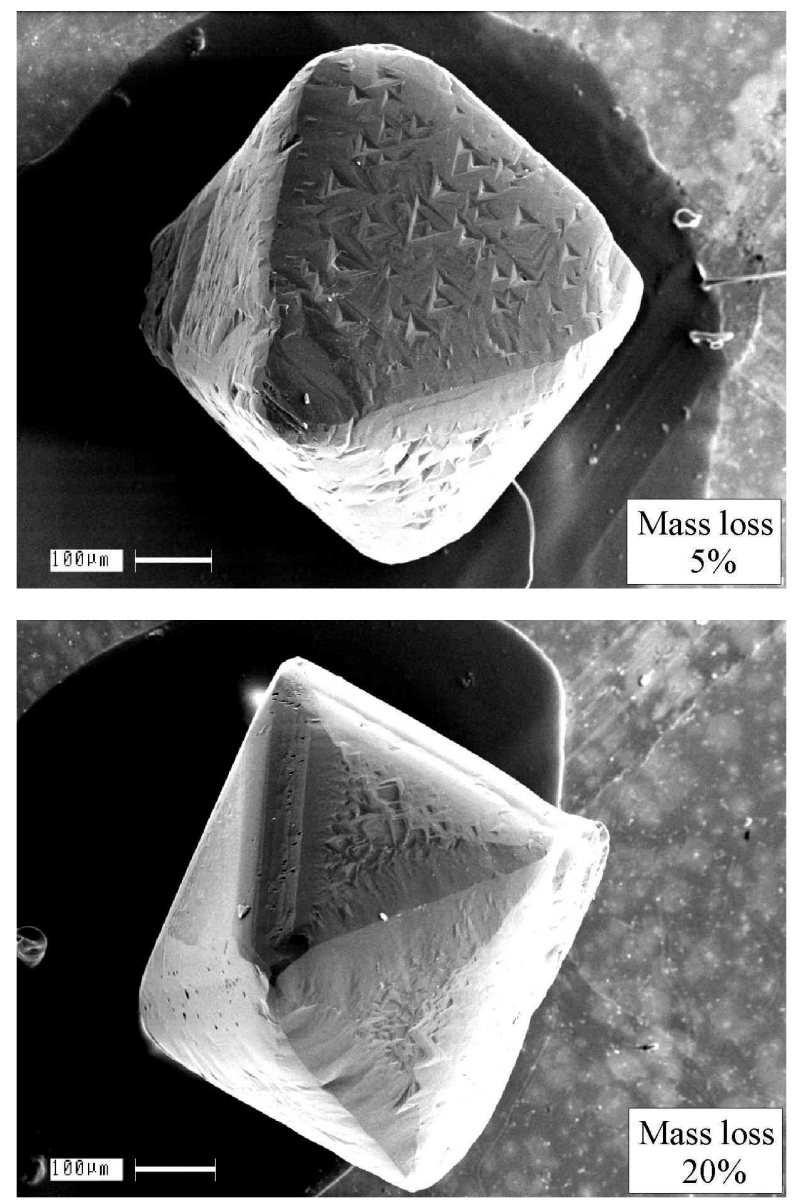

Fig. 2. SEM micrographs of dissolution forms of diamond in $\mathrm{CO}_{2}$-containing carbonate melts.
With the experimental duration up to $5 \mathrm{~h}$ and dissolution degree up to $18 \%$, numerous etch pits, positive trigons, form on the $\{111\}$ faces (Fig. 2). Besides, layer-by-layer dissolution of the $\{111\}$ faces takes place. The corners of the triangular shield-shaped layers are directed to the edges of the octahedron. As a result of dissolution, curved surfaces of trigontrioctahedroids are formed on the crystal edges. These surfaces typically exhibit linear striation and rounded triangular hillocks. With an increase in crystal weight loss to $29 \%$, the trigon-trioctahedroids surfaces completely terminate the initial $\{111\}$ faces (Fig. 2). Edges joining the $<001>$ apexes disappear and the dissolution form transforms from trigon-trioctahedroid to dodecahedroid. Rounded surfaces have fine shagreen.

\section{Dissolution of diamond in $\mathrm{H}_{2} \mathrm{O}$-containing carbonate melts}

The concentration of water in the experiments varied from 7.0 wt. $\%$ to 17.2 wt.\%. In experiments with minimal water content (7.0 wt.\%), positive trigons are formed on the $\{111\}$ faces and the edges of the crystals are truncated by narrow curved surfaces of trigontrioctahedroid. At water content of $8.0 \mathrm{wt} \%$ and higher, negative trigons and ditrigonal or shield-shaped
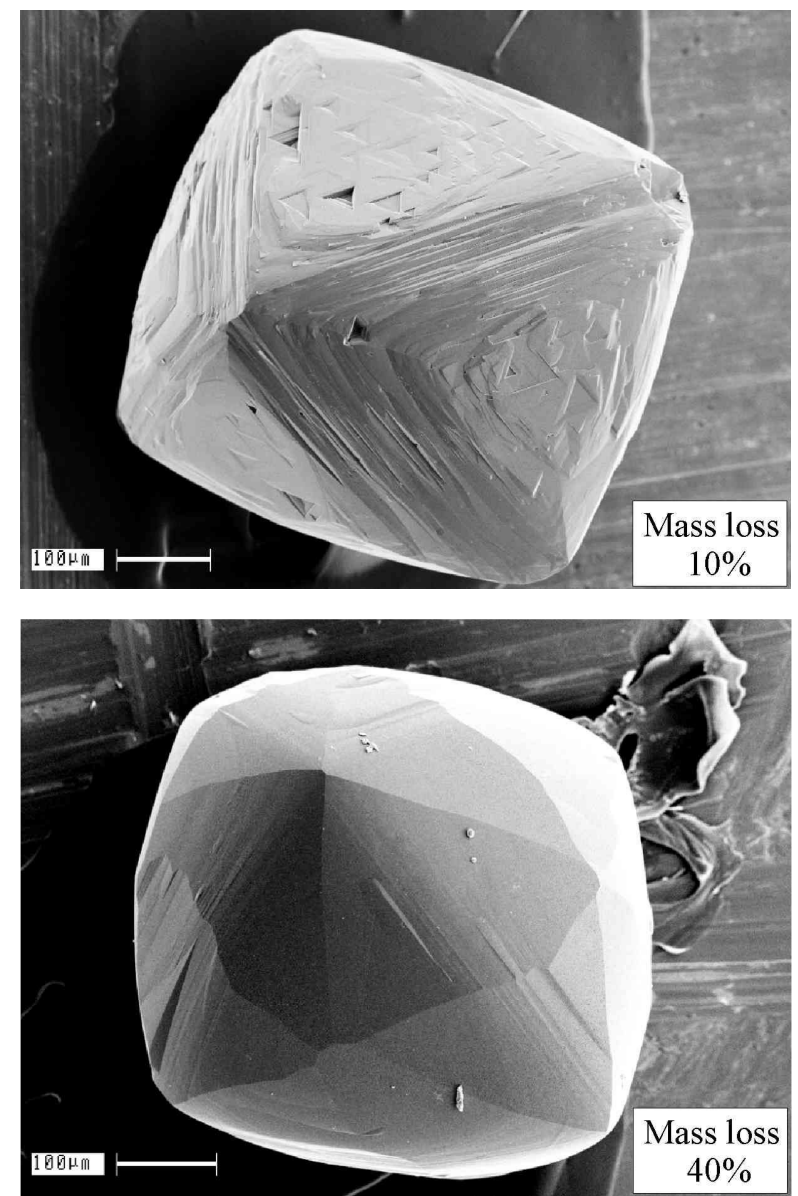

Fig. 3. SEM micrographs of dissolution forms of diamond in water-containing carbonate melts. 
plates are formed on the $\{111\}$ faces. The trigons are typically step-like and their walls make angles with the (111) face of $7.5^{\circ}, 12.5^{\circ}, 16^{\circ}$ and $20.5^{\circ}$, that correspond to the $\{443\},\{553\},\{221\}$ and $\{833\}$ faces. Shallow trigons are formed by vicinal surfaces lying at an angle of $1-2^{\circ}$ to the $\{111\}$ faces. At crystal dissolution for more than 20-25\% relative to the initial mass, the $\{111\}$ faces disappear. Newly formed surfaces of tetrahexadroids have serrate laminae and drop-like hillocks sometimes. These rounded surfaces have small curvature and the shape of the dissolution form nearly corresponds to hexaoctahedron. With an increase in the degree of crystal dissolution, the curvature of the surfaces increases and diamond crystals attain a form of rounded tetrahexahedroids, similar to that of natural rounded diamonds (Fig. 3).

The results obtained in this study show that at oxygen fugacity of 1-2 logarithmic units lower than CCO buffer, diamond actively dissolves in both volatile-free and $\mathrm{H}_{2} \mathrm{O}\left(\mathrm{CO}_{2}\right)$-containing carbonate melts in the field of thermodynamic stability of diamond. The orientation of trigons on the $\{111\}$ faces and forms of diamond dissolution depend on the amount of water in the system. In carbonate melts containing not higher than 7.0 wt. $\%$ of $\mathrm{H}_{2} \mathrm{O}$, positive trigons are formed on the $\{111\}$ diamond faces. The dissolution forms are trigontrioctahedroids or dodecahedroids depending on the degree of crystal dissolution and presence of $\mathrm{CO}_{2}$ in the melt (Fig. 4). An increase in water content in carbonate melts to more than $8.0 \mathrm{wt} . \%$ results in the formation of negative trigons on the $\{111\}$ faces. So far it has been considered that the orientation of trigons on the $\{111\}$ diamond faces is determined only by oxygen fugacity and dissolution temperature. Our experimental data on diamond dissolution at high pressure clearly show that one of the factors controlling the orientation of trigons is the $\mathrm{H}_{2} \mathrm{O} / \mathrm{CO}_{2}$ ratio in the system. Simultaneously with the change of the trigons orientation the form of diamond dissolution also changes. The dissolution forms of diamond in water containing melts are tetrahexahedroids, which are similar to natural rounded diamonds (Fig. 4).

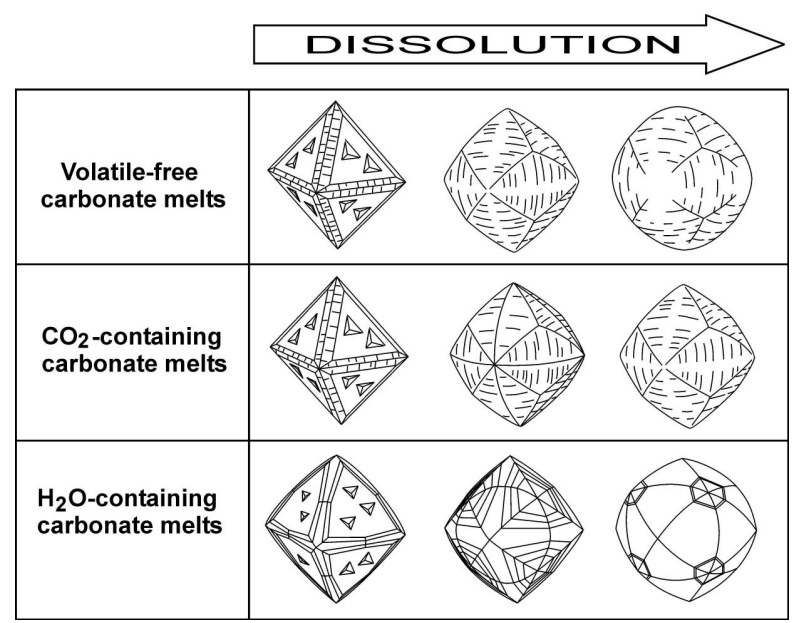

Fig. 4. Evolution scheme for the morphology of octahedral diamond crystals during dissolution in carbonate melts.
Thus, the results of the present study prove that carbonate melts are active media for diamond dissolution at the mantle P,T parameters. However, a key factor affecting the morphology of natural diamond upon its dissolution in natural carbonate and carbonatesilicate media is the presence of water in the system.

\section{References}

Fedortchouk, Y., Canil, D., Semenets, E., 2007. Mechanisms of diamond oxidation and their bearing on the fluid composition in kimberlite magmas. Am. Mineral. 92, 1200-1212.

Kanda, H., Yamaoka, S., Setaka, N., and Komatsu, H., 1977. Etching of diamond octahedrons by high pressure water. Journal of Crystal Growth, 38, 1-7.

Khokhryakov, A.F., Palyanov, Yu.N., 2000, Dissolution forms of diamond crystals in $\mathrm{CaCO}_{3}$ melt at $7 \mathrm{GPa}$. Russian Geology and Geophysics, 41, 682-687.

Khokhryakov, A.F., Palyanov, Yu.N., 2007. The evolution of diamond morphology in the process of dissolution: Experimental data. Am. Mineral. 92, 909-917.

Kozai, Y., Arima, M., 2005. Experimental study on diamond dissolution in kimberlitic and lamproitic melts at $1300-1420^{\circ} \mathrm{C}$ and $1 \mathrm{GPa}$ with controlled oxygen partial pressure. Am. Mineral. 90, 1759-1766.

Pal'yanov, Yu.N., Sokol, A.G., Borzdov, Yu.M., Khokhryakov, A.F., 2002. Fluid-bearing alkalinecarbonate melts as the medium for the formation of diamonds in the Earth's mantle: an experimental study. Lithos 60, 145-159.

Sokol, A.G., Borzdov, Yu.M., Pal'yanov, Yu.N., Khokhryakov, A.F., Sobolev, N.V., 2001. An experimental demonstration of diamond formation in the dolomite-carbon and dolomite fluid-carbon systems. Eur. J. Mineral. 13 (5), 893-900. 